\title{
Cognitive Assessment in Multiple Sclerosis
}

\author{
An Expert Interview with Dawn Langdon \\ Royal Holloway, University of London, London, UK
}

DOl: https://doi.org/10.17925/ENR.2018.13.1.12

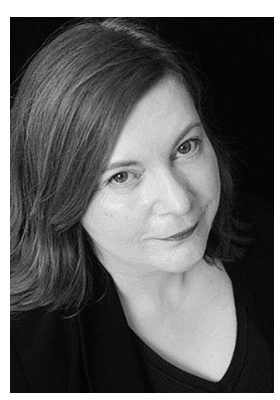

\section{Dawn Langdon}

Dawn Langdon is Professor of Neuropsychology at Royal Holloway, University of London. Her work centres on cognitive aspects of multiple sclerosis (MS) and her research interests include developing the Motor Planning Index, a clinical measure of cerebellar function, and investigating how cognition affects employment for people with MS. She is Co-Chair of Brief International Cognitive Assessment for MS (BICAMS), an international project to facilitate cognitive assessment and management (WwW.BICAMS.net). She is also Co-Chair of MS in the 21st Century (www. msinthe21stcentury.com), a project involving people with MS and healthcare professionals to promote communication and improve healthcare. She has led the development of the BRIMMS protocol (Benefits and Risks of Medication for Multiple Sclerosis), a format which optimally presents drug information to people with MS. Professor Langdon has been cognitive lead on a number of multinational trials for industry, including Merck's ongoing CLARIFY phase IV trial of quality of life and cognition. She is neuropsychology lead on the Trajectories of Outcome in Neurological Conditions (TONiC), a national study examining the factors that influence quality of life in patients with neurological conditions (https://tonic.thewaltoncentre. nhs. UK/). She is also a Trustee of the UK MS Trust, having authored their online cognition tool for people with MS (www.stayingsmart.org.uk). She is a frequent contributor to international meetings and advisory boards and has published widely.

\section{Keywords}

Multiple sclerosis, cognitive assessment, BICAMS, cognition

Disclosure: Dawn Langdon has participated in speaker bureaux for Bayer, Merck, Almirall, Execemed, TEVA, Roche, Novartis, Biogen and Sanofi; has had consultancy fees from Novartis, Bayer, Merck, Biogen, TEVA and Sanofi; and has had research grants from Bayer, Merck, Novartis and Biogen. All are paid into Dawn Langdon's institution.

Review Process: This is an expert interview and as such has not undergone the journal's standard peer review process.

Authorship: The named author meets the International Committee of Medical Journal Editors (ICMJE) criteria for authorship of this manuscript, takes responsibility for the integrity of the work as a whole, and has given final approval for the version to be published.

Open Access: This article is published under the Creative Commons Attribution Noncommercial License, which permits any non-commercial use, distribution, adaptation and reproduction provided the original author and source are given appropriate credit. (c) The Author 2018.

Received: 16 May 2018

Published Online: 6 June 2018

Citation: European Neurological Review. 2018;13(1):12-3 Corresponding Author: Dawn Langdon, Professor of Neuropsychology, Royal Holloway University of London, Egham, Surrey, TW20 OEX, UK.

E:d.langdon@rhul.ac.uk Twitter: @Dawnlangdon

Support: No funding was received in

the publication of this article.
$\mathrm{M}$ ultiple sclerosis (MS) is a debilitating neurodegenerative disease with marked effects on cognitive function. Assessing the cognitive changes experienced by patients with MS requires specific, tailored tools. In an expert interview, Professor Dawn Langdon explains cognitive changes in MS as well as the background, benefits and process of the Brief International Cognitive Assessment for MS (BICAMS), a test designed for international use that is brief and effective in assessing cognitive function in MS.

\section{Q. What are the main cognitive changes experienced by patients with MS?}

The most severe and prevalent cognitive deficit at all stages of the disease is slowed information processing speed. ${ }^{1}$ This can be thought of as 'reduced bandwidth'. It means that people with MS find it harder to multitask and are easily distracted. For example, using a sat nav with spoken route directions may cause difficulty whilst they are driving. Also, people with MS working in a call centre with the task of listening to and processing a lot of spoken information may struggle. The next most severe and prevalent cognitive deficits are in visual and verbal memory. This is especially true of prospective memory, which might involve remembering to do something you had previously planned to do, as well as recalling the planned action at a future point in time. This can cause problems in relationships if the partner is not fully aware of this symptom of MS. An example could revolve around a person with MS who is no longer working, and a working partner who requests that some minor chores be done during the day. They might return home to find that the tasks have not been completed, which could be interpreted as the person with MS being unwilling to help around the house, or not appreciating the efforts of their working partner. Executive functions are also affected, which means that planning, organisation and problem-solving skills are diminished.

\section{Q. Which areas of cognition are examined in BICAMS?}

The Brief International Cognitive Assessment for MS (BICAMS) assesses information processing speed, immediate verbal recall memory and immediate visual recall memory. ${ }^{2}$

\section{Q. How is the BICAMS test performed?}

BICAMS takes 15 minutes to complete and requires some paper tests, three sheets of A4 blank paper and a pencil with an eraser. The Symbol Digit Modalities Test (SDMT) was selected to measure information processing speed. The test consists of single digits, each paired with a particular abstract symbol. The person with MS is presented with a key that matches nine digits to an abstract shape, and rows of the nine symbols that are arranged pseudo-randomly without paired numbers. They must say the numbers that go with each symbol in turn. The SDMT can be completed within 5 minutes, including the delivery of instructions, and time allocated for practice and testing. In the California Verbal Learning Test-II (CVLT-II), the test of verbal recall, patients are 
read a list of words at a slightly slower rate than one item per second, and asked to recall as many items as possible in any order, for each of the five trials. The test is a 16-item word list, with four items belonging to each of four categories, arranged randomly. The Brief Visual Memory Test-Revised (BVMT-R), the test of visual recall, requires the person with MS to inspect a $2 \times 3$ stimulus array of abstract geometric figures. There are three learning trials of 10 seconds each. After 10 seconds, the array is hidden from view, and the person with MS is required to draw the geometric figures in the correct position from memory, i.e. exactly reproduce the array.

\section{Q. What are the advantages of BICAMS compared with existing methods?}

a) BICAMS takes 15 minutes to administer and it has the same sensitivity to cognitive impairment in MS as the recommended, 'gold standard' minimal assessment of cognitive function in multiple sclerosis (MACFIMS) battery, which takes 90 minutes and requires a number of expensive and cumbersome cognitive tests. ${ }^{3}$

b) BICAMS can be administered by most healthcare professionals, whereas other batteries, or a bespoke assessment, must be administered by a qualified neuropsychologist. c) BICAMS has been embraced by the international MS community, with a published international validation protocol. ${ }^{4}$ Currently there are 30 countries in the international validation pipeline, of which 14 have published so far. These national validations mean that an increasing number of countries can use BICAMS, confident that it is effective and appropriate for their language and culture.

d) Over 20 scientific studies have been published using BICAMS, which means that BICAMS scores can be related to, for example, the effectiveness of medication or physical exercise interventions.

\section{Q. What evidence is available regarding the validation of BICAMS?}

There are currently 30 countries in the international validation pipeline. The original committee paper recommending BICAMS is open access and outlines the methodology and justification for the tests selected. ${ }^{.}$So far, 14 countries have published BICAMS national validations (America, Argentina, ${ }^{5}$ Belgium, ${ }^{6}$ Brazil, Canada, ${ }^{8}$ Czech Republic, ${ }^{3}$ Greece, Hungary, ${ }^{10}$ Iran, ${ }^{11}$ Ireland, ${ }^{12}$ Italy, ${ }^{13}$ Japan, ${ }^{14}$ Lithuania15 and Turkey ${ }^{16}$. Amalgamating national validation data has allowed regression models to be built with larger datasets. ${ }^{17}$ An iPad BICAMS has also been developed and is undergoing preliminary validation.
1. Benedict RHB, DeLuca J, Enzinger C, et al. Neuropsychology of multiple sclerosis: looking back and moving forward. J Int Neuropsychol Soc. 2017;23:832-42.

2. Langdon $\mathrm{D}$, Amato M, Boringa J, et al. Recommendations for a Brief In, A (BICAMS). Mult Scler J.2012:18:891-8. (BICAMS). Mult SCler J. 2012,18:891-8.

3. Dusankova JB, Kalincik T, Havrdova E, Benedict RHBB. Cross cultural validation of the minimal assessment of cognitive function in multiple sclerosis (MACFIMS) and the brief international cognitive assessment for multiple sclerosis (BICAMS). Clin Neuropsychol. 2012;26:1186-200.

4. Benedict RH, Amato MMP, Boringa JJ, et al. Brief International Cognitive Assessment for MS (BICAMS): international standards for validation. BMC Neurol. 2012;12:55.

5. Vanotti S, Smerbeck A, Benedict RH, Caceres F. A new assessment tool for patients with multiple sclerosis from Spanish-speaking countries: validation of the Brief International Cognitive Assessment for MS (BICAMS) in Argentina. Clin Neuropsychol. 2016;30:1023-31.

6. Costers L, Gielen J, Eelen PL, et al. Does including the full CVLT-II and BVMT-R improve BICAMS? Evidence from a Belgian (Dutch) validation study. Mult Scler Relat Disord. 2017;18:33-40.
7. Spedo CT, Frndak SE, Marques VD, et al. Cross-cultural adaptation, reliability, and validity of the BICAMS in Brazi. Clin Neuropsychol. 2015;29:836-46.

8. Walker LAS, Osman L, Berard JA, et al. Brief Internationa cognitive Assessment for Multiple Sclerosis (BICAMS): Canadian contribution to the international validation project. J Neurol Sci. 2016;362:147-52.

9. Polychroniadou E, Bakirtzis C, Langdon D, et al. Validation of the Brief International Cognitive Assessment for Multiple Sclerosis (BICAMS) in Greek population with multiple sclerosis. Mult Scler Relat Disord. 2016;9:68-72

10. Sandi D, Rudisch T, Füvesi J, et al. The Hungarian validation of the Brief International Cognitive Assessment for Multiple Sclerosis (BICAMS) battery and the correlation of cognitive impairment with fatigue and quality of life. Mult Scler Relat Disord. 2015;4:499-504.

11. Eshaghi A, Riyahi-Alam S, Roostaei $T$, et al. Validity and reliability of a Persian translation of the Minimal Assessment of Cognitive Function in Multiple Sclerosis (MACFIMS). Clin Neuropsychol. 2012:26:975-84.

12. O'Connell $K$, Langdon $D$, Tubridy $N$, et al. A preliminary validation of the brief international cognitive assessment for multiple sclerosis (BICAMS) tool in an Irish population with multiple sclerosis (MS). Mult Scler Relat Disord. 2015;4:521-5.

13. Goretti B, Niccolai C, Hakiki B, et al. The Brief International Cognitive Assessment for Multiple Sclerosis (BICAMS): normative values with gender, age and education corrections in the Italian population. BMC Neurol. 2014;14:171.

14. Nino M, Fukazawa T, Kira JI, et al. Validation of the Brief International Cognitive Assessment for Multiple Sclerosis in Japan. Mult Scler J Exp Transl Clin. 2017;26;3:2055217317748972

15. Giedraitienè N, Kizlaitienè R, Kaubrys G. The BICAMS battery for assessment of Lithuanian-speaking multiple sclerosis patients: relationship with age, education, disease disability, and duration. Med Sci Monit. 2015;10:3853-9.

16. Ozakbas S, Yigit P, Cinar BP, et al. The Turkish validation of the Brief International Cognitive Assessment for Multiple Sclerosis (BICAMS) battery. BMC Neurol. 2017;17:208.

17. Smerbeck $A$, Benedict RHB, Eshaghi $A$, et al. Influence of nationality on the Brief International Cognitive Assessment for Multiple Sclerosis (BICAMS). Clin Neuropsychol. 2018;32:54-62. 